|| Print ISSN: 2589-7837 || Online ISSN: 2581-3935 ||

International Journal of Medical Science and Diagnosis Research (IJMSDR)

Available Online at www.ijmsdr.com

NLM (National Library of Medicine ID: 101738824)

\title{
Coexistence of Mycobacterium Tuberculosis and Mycobacterium Leprae in a Patient- an Alarm of Inadequate Innate Immune Response?
}

\author{
Dr. A. Ramesh ${ }^{1}$, Dr. Rajkumar Kannan ${ }^{2}$, Dr. Samuel Jeyaraj Daniel ${ }^{3}$, \\ Dr. Vishnupriya $\mathbf{G}^{4}$ \\ ${ }^{1}$ MD., DD., DNB (DVL), Professor, Department of DVL, Madras Medical College \\ ${ }^{2}$ MD DD, Associate Professor, Department of DVL, Madras Medical College \\ ${ }^{3}$ MD, Associate Professor, Department of DVL, Madras Medical College \\ ${ }^{4}$ MBBS, Postgraduate student, Department of DVL, Madras Medical College
}

\section{Conflicts of Interest: Nil}

Corresponding author: Dr Vishnupriya G

DOI: https://doi.org/10.32553/ijmsdr.v5i8.836

\begin{abstract}
:
Mycobacterium tuberculosis (M.Tb) and Mycobacterium leprae (M.Leprae) are Gram-positive aerobic Acid-Fast Bacilli (AFB) causing chronic granulomatous infections like Tuberculosis (TB) and Leprosy respectively. They can present with varied manifestations according to the host's immune status and response ${ }^{[1]}$ Both are prevalent in clusters in developing countries; however, the simultaneous occurrence of both infections in an individual is rare even in endemic areas $(0.02$ per 100,000 population $){ }^{[2]} \mathrm{Few}$ case reports showing the coexistence of TB and leprosy, majority are TB developing following immunosuppressive treatment of leprosy reactions. Of which pure neuritic presentation of leprosy in an old treated case of Pulmonary Tuberculosis (PTB) has not been reported yet. Here we are reporting a case of PTB while on treatment developed neurological manifestations, diagnosed to be a case of poly neuritic leprosy (PNL). PNL is a rare form of leprosy, characterised by an area of sensory loss along the distribution of single/multiple thickened nerve trunks with or without motor deficit in the absence of skin lesions.
\end{abstract}

Keywords: TB with Pure Neuritic Hansen, SNP- PPTN22, Defective Lymphocyte Transformation, SNP- Granulomatous infection

\section{Introduction:}

Polyneuritic leprosy (PNL) is characterized by an area of sensory loss along the distribution of an involved nerve trunk with or without motor deficit, in the absence of skin patch. According to Indian studies, PNL constitutes about $4-18 \%$ of leprosy patients. It is reportedly higher in South India comprising up to $18 \%$ of new cases. It is more common in men and most common in the $15-30$ age group. ${ }^{[3]}$ Patient usually presents with weakness and numbness, neuropathic pain, grossly thickened nerves or nerve abscesses. In PNL, upper limb nerves are more commonly involved, ulnar nerve being the most common followed by lateral popliteal nerve in the lower limb. However, any nerve trunk or cutaneous nerve can be involved. ${ }^{[4]}$

\section{Case Report}

A 50 year old male, driver by occupation came to our OPD with complaints of difficulty in using left hand and right foot for the past two years associated with numbness. He was apparently normal three years ago, upon evaluation for chronic cough, he was diagnosed to be a case 
PTB (fig 1) and started on standard first line Anti Tuberculosis Treatment (ATT) regimen. Four months following which patient developed weakness and numbness and he defaulted treatment. He is a Known case of type 2 diabetes mellitus on treatment. Non smoker and non alcoholic. There was no family history of PTB/Leprosy.

The examination findings were consistent with left ulnar and right lateral popliteal sensorimotor neuropathy (TABLE 1).

Table 1: Motor System Examination

\begin{tabular}{|l|l|l|l|l|}
\hline & Right Upper Limb & Left Upper Limb & Right Lower Limb & Left Lower Limb \\
\hline Tone & Normal & Reduced & Reduced & Normal \\
\hline Bulk & Normal & Reduced & Reduced & Normal \\
\hline Power & Normal & $\begin{array}{l}3 / 5 \\
\text { (Small Muscles Of Hand) }\end{array}$ & $\begin{array}{l}3 / 5 \\
\text { (Small Muscles Of Foot) }\end{array}$ & \\
\end{tabular}

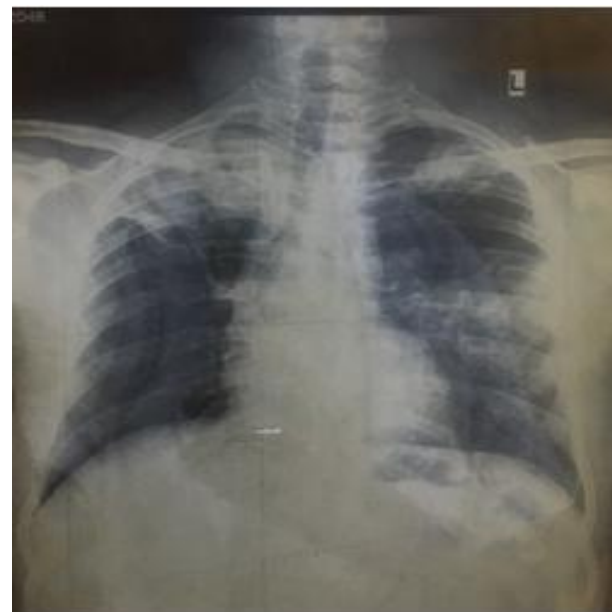

Figure 1 - Chest Xray PA view taken before ATT showing, active consolidatory foci over the left middle lobe, cavitatory lesions over the left upper lobe.

There was marked clawing and guttering of left hand (fig 2) and right foot drop and clawing of toes (fig 3). There were no signs of neuritis clinically. A single trophic ulcer was noted over the lateral malleolus of right leg (fig 4).
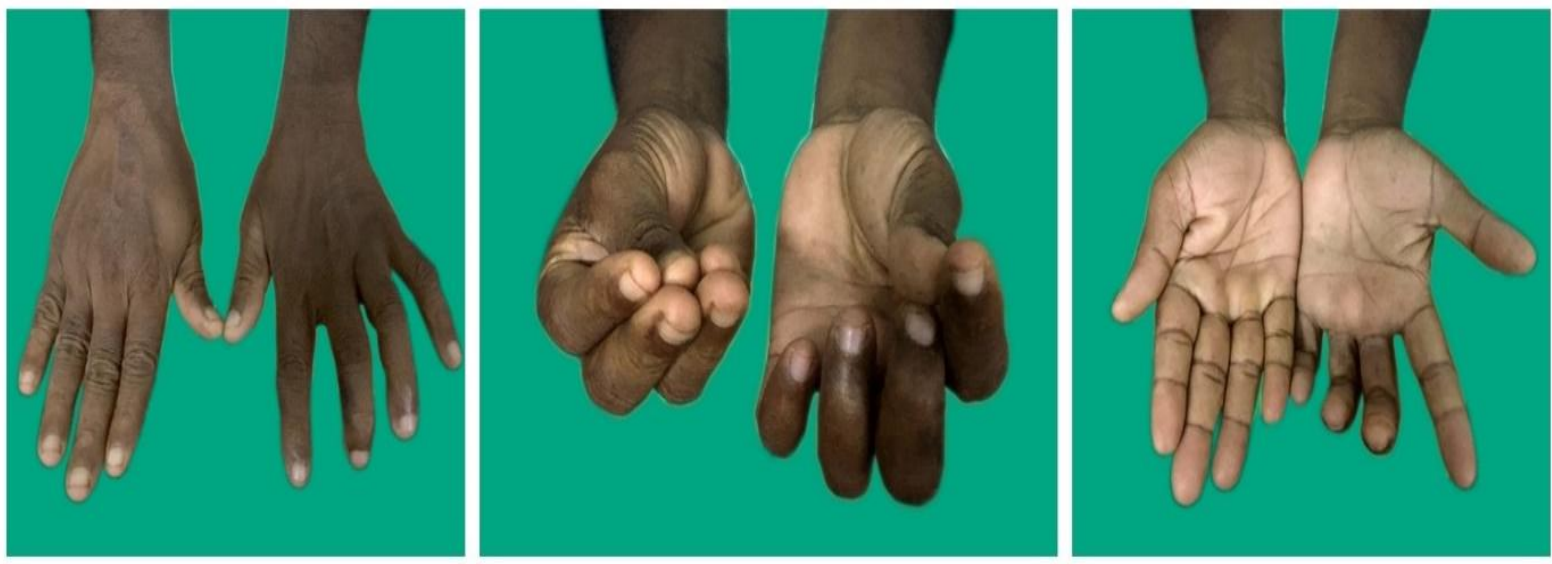

Figure 2: left hand-partial claw hand with guttering and wasting 


\begin{tabular}{|l|l|}
\hline A. Ramesh et al & International Journal of Medical Science and Diagnosis Research (IJMSDR) \\
\hline
\end{tabular}
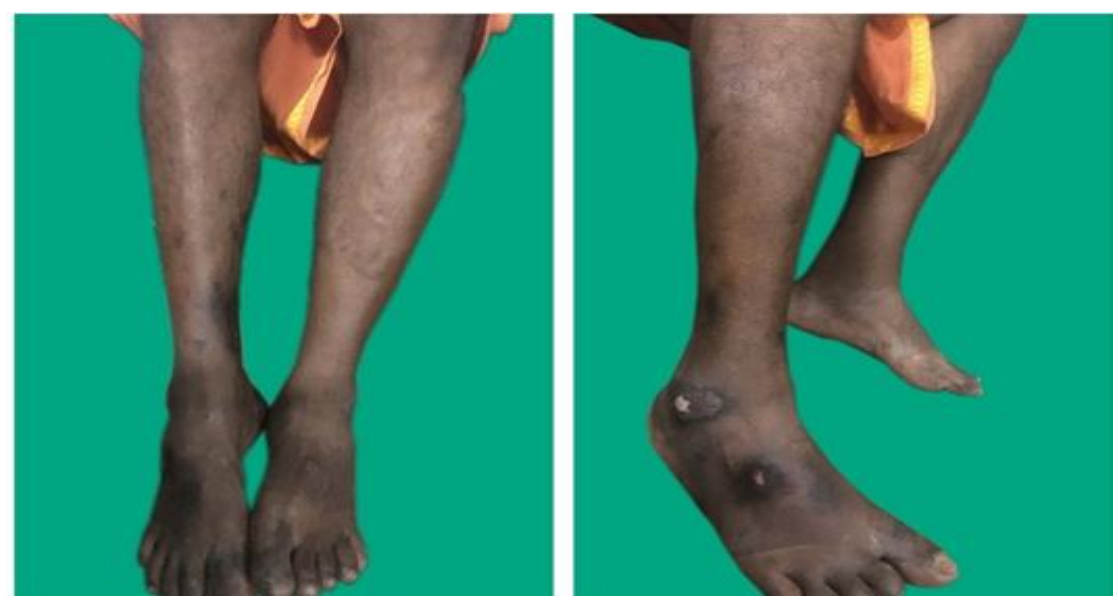

Figure 3: right foot drop with trophic ulcer over the lateral malleolus
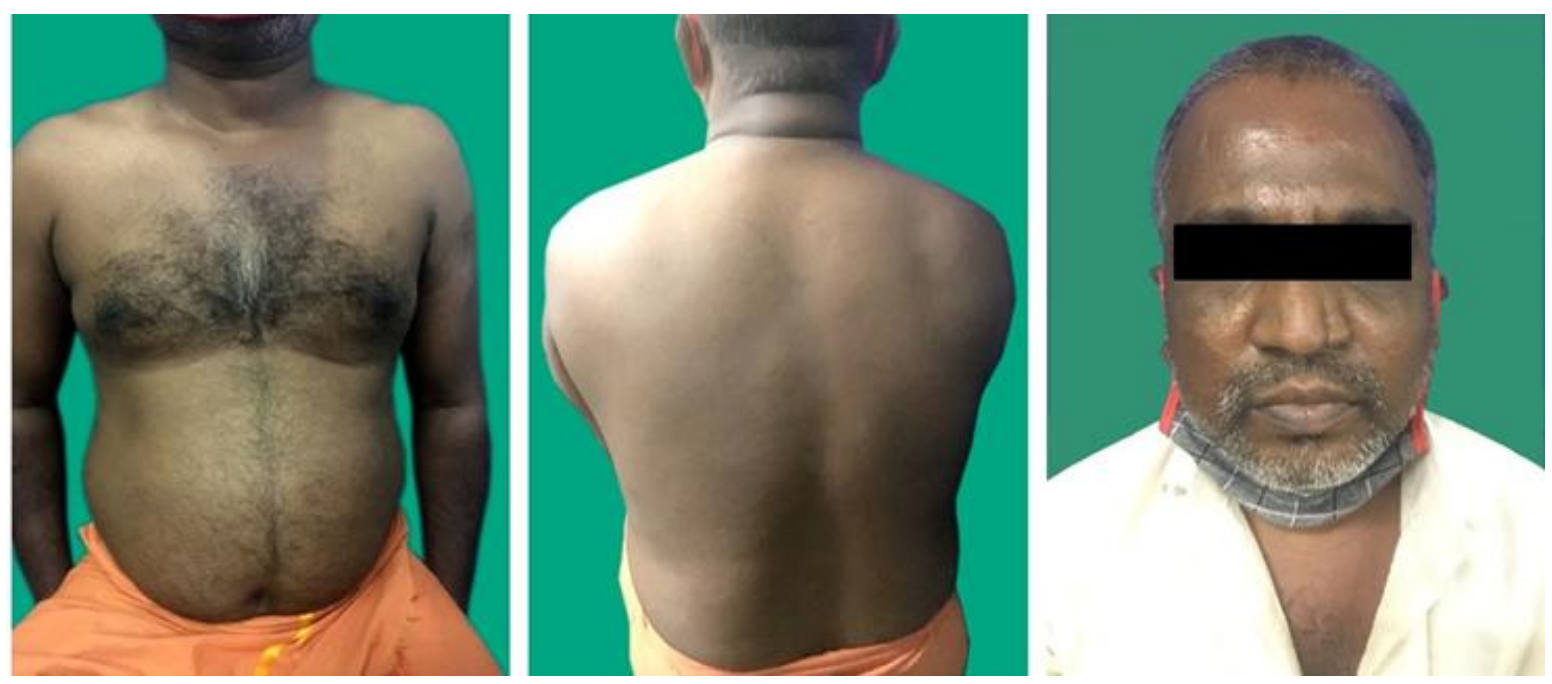

Figure 4: Absence of hypopigmented patches in the trunk, chest, face

No hypopigmented/erythematous patch anywhere in the body (fig 4). Ocular, genital, spine examination appeared normal. A provisional diagnosis of mononeuritis presentation of PNL was made and investigated for the same.

Following neurological evaluation, Nerve Conduction Study (NCS) (TABLE 2) showed features suggestive of mononeuritis multiplexa.

Table 2: Nerve Conduction Study Reports Sensory Nerve Studies

\begin{tabular}{|l|l|l|l|l|}
\hline \multicolumn{2}{|c|}{} & Latency & Amplitude & Nerve conduction velocity \\
\hline Median & RT & 3.17 & 10.7 & 39.43 \\
\hline Median & LT & 2.00 & 3.9 & 60.00 \\
\hline Ulnar & RT & 2.25 & 11.8 & 48.89 \\
\hline Ulnar & LT & 3.58 & 0.6 & 33.52 \\
\hline Sural & RT & 3.63 & 0.2 & 30.39 \\
\hline Sural & Lt & 1.83 & 5.2 & 60.11 \\
\hline
\end{tabular}


Motor Nerve Studies

\begin{tabular}{|l|l|l|l|l|l|l|}
\hline & Latency & Amplitude & NCV & FMN \\
\hline Median RT & 3.13 & 9.27 & 18.2 & 12.40 & 40.7 & 30.6 \\
\hline Median LT & 3.02 & 9.27 & 12.9 & 9.00 & 38.4 & 36.0 \\
\hline Ulnar RT & 1.88 & 7.19 & 7.1 & 6.40 & 49.0 & 28.8 \\
\hline Ulnar LT & Absent & \multicolumn{3}{l}{} \\
\hline Peroneal RT & Absent & \multicolumn{5}{l|}{} \\
\hline Peroneal LT & 3.23 & 12.60 & 2.1 & 1.20 & 39.5 & Absent \\
\hline Tibial RT & 5.52 & 16.56 & 2.1 & 0.70 & 36.2 & 64.8 \\
\hline Tibial LT & 3.96 & 15.00 & 3.7 & 2.30 & 37.6 & 57.1 \\
\hline
\end{tabular}

Impression - Decrease in linear conduction velocity with increase in the latency of left ulnar nerve and right peroneal nerve noted. Features suggestive of LT Ulnar (Motor, Sensory), LT Median sensory, RT Peroneal, RT Tibial, and RT Sural Neuropathy. [LT- left, RT - right, NCV - Nerve Conduction Velocity].

High frequency ultrasonography (HRUS) of the nerve showed- thickened hypoechoic left ulnar nerve, left median nerve, right posterior tibial nerve, bilateral lateral popliteal nerve with increased vascularity. Slit skin smear of bilateral ear lobules was negative. A high resolution Computerized tomography (HRCT) (fig 5) showed features suggestive of old PTB sequelae.

Confirming the evidence of pulmonary tuberculosis in the patient. Fiber optic bronchoscopy with gene Xpert studies, done to rule out active tuberculosis, turned out to be negative. After obtaining opinion and clearance from Neurologist and Pulmonologist, patient was diagnosed to be a case of old pulmonary tuberculosis now presenting as PNL. Patient was started on a fixed drug combination of MultiDrug Therapy (MDT) as per WHO regime for multibacillary leprosy for twelve months after routine laboratory workup and followed up. Also patient was put on metered dose inhalers of low dose corticosteroids with bronchodilators for the bronchiectatic changes.

\section{Discussion}

PNL can display almost the complete spectrum of Ridley -Jopling classification, of which borderline tuberculoid (BT) being the most frequent. Mononeuritis multiplex which is involvement of multiple unrelated and distant nerve trunks is the most common presentation. In a large study on PNL from India, it was noted that $26 \%$ were mononeuritic, while in $39 \%$ of cases multiplexa presentation was seen. ${ }^{[5]}$ Our patient presented with a left ulnar nerve and right lateral popliteal nerve involvement, with probable mononeuritic multiplexa like presentation.

Diagnosis is mainly clinical accompanied by NCS which acts as a sensitive tool to detect marked slowing of conduction in leprosy-affected nerves, decreased amplitude of sensory-motor potentials, ${ }^{[5]}$ and changes in latency. HRUS frequency (15-20 MHz) along with colour doppler study is used effectively in detecting increased blood flow signals of endoneural and perineural vessels, are mainly useful during reactional phases of the disease. The vascular changes in HRUS were correlated with edema and vascularity histologically, making it an useful tool to detect neuritis. ${ }^{[6]}$ The gold standard for confirmation of PNL is by nerve biopsy. However, diagnostic intraneural epitheloid granulomas are seen only in few cases.

Old evidence of PTB in this patient indicates a state of immunosuppression, which makes the patient susceptible to other granulomatous infections due to several pathogenic mechanisms. Leprosy and PTB coinfection in the same patient was first reported in $1954 .{ }^{[7]}$ The coinfection of tuberculosis and leprosy depends on sharing of epidemiological factors such as overcrowding, 
malnutrition, improper sanitation, and low socioeconomic status. Single-Nucleotide Polymorphisms (SNP) rs2476601, involving PTPN22 [protein tyrosine phosphatase nonreceptor type 22]-C1858T predisposes to both M.Tb and M.leprae infection. ${ }^{[8]}$ Taking into account the prolonged incubation period of M.leprae, which varies from few weeks to 30 years (average is 4.4 years in South India) and the generation time (doubling time) - 11 to 13 days, it is rather difficult to understand the innate immune mechanism response of the host in Hansen's disease. The role of Toll Like Receptors(TLR) proteins TLR2/1 heterodimer and TLR 4 are important points of recognition and activation of antimicrobial and phagocytic cascade. Also the Ctype lectin receptors on mature macrophages bind the mannose-capped lipoarabinomannan on $M . T b$ and M.leprae and influence phagocytosis and TNF alpha production. ${ }^{[9]}$ Any defect in this innate immune response against the Mycobacterium complex can predispose an individual to varying granulomatous diseases. However PNL, with absent skin involvement and rare systemic involvement in multibacillary cases has rarely been associated with M.Tb infection. Hence this case is being reported for the rare association of $M . T b$ with PNL. Also as discussed above, it is the treatment of leprosy reactions with immunosuppressants predisposes to M.tb. Per contra this patient has acquired $M . t b$ as a primary infection and PNL later in the time scale which rules out the possibility of immunosuppression as a predisposing factor to M.leprae.

\section{References}

1. Keragala BS, Herath HM, Janapriya GH, Vanitha S, Balendran T, Janani T, Keragala TS, Gunasekera CN. Coexistence of mycobacterial infections-Mycobacterium tuberculosis and Mycobacterium leprae-in Sri Lanka: a case series. Journal of Medical Case Reports. 2020 Dec;14(1):1-8.
2. Rawson TM, Anjum V, Hodgson J, Rao AK, Murthy K, Rao PS, Subbanna J, Rao PV. Leprosy and tuberculosis concomitant infection: a poorly understood, age-old relationship. Lepr Rev. 2014 Dec 1;85(4):288-95.

3. Mendiratta V, Khan A, Jain A. Primary neuritic leprosy: a reappraisal at a tertiary care hospital. Indian journal of leprosy. 2006 Jul 1;78(3):261-7.

4. Rao PN, Suneetha S. Pure neuritic leprosy: Current status and relevance. Indian Journal of Dermatology, Venereology, and Leprology. 2016 May 1;82(3):252.

5. Goulart IM, Goulart LR. Leprosy: diagnostic and control challenges for a worldwide disease. Archives of dermatological research. 2008 Jul;300(6): 269-90.

6. Elias Jr J, Nogueira-Barbosa MH, Feltrin LT, Furini RB, Foss NT, Marques Jr W, dos Santos AC. Role of ulnar nerve sonography in leprosy neuropathy with electrophysiologic correlation. Journal of Ultrasound in Medicine. 2009 Sep;28 (9):1201-9.

7. Relvich AL. The treatment of tuberculosis in leprosy patients. Lepr Rev. 1954 Oct;25(4):179-86.

8. Li S, Wang X, Zhao Y, Yang J, Cui T, Zhao ZJ, Chen Y, Zheng Z. Association of PTPN22-C1858T Polymorphism With Susceptibility to Mycobacterium tuberculosis and Mycobacterium leprae Infection: A Meta-Analysis. Frontiers in immunology. 2021;12.

9. Chatterjee D, Roberts AD, Lowell K, Brennan PJ, Orme IM. Structural basis of capacity of lipoarabinomannan to induce secretion of tumor necrosis factor. Infection and immunity. 1992 Mar;60(3):1249-53. 